\title{
Heteroduplex analysis of T-cell receptor $\gamma$ gene rearrangement as an adjuvant diagnostic tool in skin biopsies for erythroderma
}

Background: Erythroderma, defined as red skin covering most of the body surface often accompanied or followed by exfoliation, is the clinical manifestation of at least six different underlying etiologies with allergic or irritant contact dermatitis, atopic/asteotic dermatitis, pityriasis rubra pilaris $(\mathrm{PRP})$, psoriasis, and seborrheic dermatitis accounting for the majority of cases. Approximately $10 \%$ of cases are due to adverse drug reactions with roughly another $10 \%$ due to cutaneous T-cell lymphoma (CTCL), predominantly mycosis fungoides, or leukemia. It is clear from multiple studies that the clinical diagnosis of the underlying entity is often difficult, as these diseases can present in a very similar fashion. A skin biopsy is usually employed in this setting as a diagnostic tool. However, the histopathologic diagnosis of the underlying cause is complicated by the subtlety of the distinguishing histologic features. In this situation, an ancillary technique demonstrating the presence of a monoclonal T-cell proliferation could help to rule in or out CTCL in cases that clinically and histopathologically do not allow a definitive diagnosis.

Methods: We retrospectively studied 25 biopsies from sixteen patients who presented to the Stanford Dermatology Clinic with erythroderma. We examined the specimens morphologically and analyzed the gamma chain of the T-cell receptor (TCR- $\gamma$ ) by polymerase chain reaction (PCR) followed by heteroduplex analysis for clonality.

We then correlated the results of our PCR and heteroduplex analyses with the patients' clinical outcomes.

Results: Four biopsies, from three patients, contained clonal TCR- $\gamma$ rearrangements; the four biopsies, all of which were equivocal histologically, correlated to diagnoses of mycosis fungoides (MF) or Sézary syndrome (SS). Twenty-one biopsies contained polyclonal T-cell populations. Eighteen of these biopsies represent patients with inflammatory dermatoses. Three of these biopsies, all of which were taken from a single patient, correlate to a diagnosis of MF.

Conclusion: TCR- $\gamma$ PGR heteroduplex analysis seems to represent an important adjuvant diagnostic tool that, used in conjunction with histopathology and clinical history, could help to clarify the underlying etiology of erythroderma.

Cherny S, Mraz S, Su L, Harvell J, Kohler S. Heteroduplex analysis of T-cell receptor $\gamma$ gene rearrangement as an adjuvant diagnostic tool in skin biopsies for erythroderma.

J Cutan Pathol 2001; 28: 351-355. (C) Munksgaard 2001.
Sarah Cherny ${ }^{1}$, Serena Mraz $^{2}$, Lyndon $\mathrm{Su}^{3}$, Jeff Harvell, ${ }^{1,2}$ and Sabine Kohler ${ }^{1,2}$

Departments of ${ }^{1}$ Pathology and ${ }^{2}$ Dermatology, Stanford University Medical Center, Stanford California, USA, Departments of ${ }^{3}$ Pathology and Dermatology, University of Michigan, Ann Arbor, Michigan, USA
Sabine Kohler, Dept. Pathology, Stanford University Medical Center, Stanford, CA 94305, USA

e-mail: skohler@stanford.edu

Accepted January 2, 2001 


\section{Cherny et al.}

Erythroderma, defined as generalized erythema involving at least $90 \%$ of the total body surface area and often accompanied by scaling, occurs in association with a variety of disorders. ${ }^{1}$ History of a preexisting dermatosis such as psoriasis, pityriasis rubra pilaris (PRP), or atopic dermatitis may aid in identifying an underlying cause in some patients. However, in most cases, identification of the underlying etiology is difficult since the histopathologic features that normally distinguish among the different dermatoses are more subtle in the context of erythroderma. ${ }^{3-5}$ Erythroderma may be the first manifestation of mycosis fungoides or Sézary syndrome (SS), which is a triad of erythrodermic MF, circulating Sézary cells in the peripheral blood $(>5-10 \%)$, and clinically enlarged lymph nodes. Well-defined histologic criteria exist for patch and plaque stage MF, but erythrodermic MF can be more difficult to recognize histologically. ${ }^{6,7}$

While Southern blotting is the gold standard to determine T-cell receptor (TCR) clonality in skin biopsies, this procedure has important disadvantages that have made polymerase chain reaction (PCR)-based methods a preferred alternative. Southern blotting requires a large amount of high quality DNA that can only be extracted from unfixed tissue, involves exposure to radioactive isotopes, and is only able to discern clonal T-cell populations that comprise at least $5 \%$ of the total T-cell population. In contrast, the PCR-based methods are comparatively fast and simple, can be performed on small quantities of DNA derived from either fresh or paraffin-embedded tissue samples, and can detect clonal populations that make up less than $1 \%$ of the T-cells in the sample. ${ }^{3}$

While multiple other studies have shown the utility of PGR analysis of the TCR as a tool in detecting a clonal population of T-cells in skin biopsies, ${ }^{3,8-11}$ none to our knowledge has approached the topic in a retrospective fashion, for the specific disease entity of erythroderma. This is important not only for determining the procedure's ability to detect clonality in the early stages of erythroderma but also for quantifying the false-positive rate among the inflammatory dermatoses.

\section{Material and methods}

\section{Patients}

We obtained 25 formalin-fixed, paraffin-embedded tissue samples from sixteen patients, all of whom presented with erythroderma to the Stanford Dermatology Clinic. Nine men and seven women were included in the study, ranging in age from 54 to 86 years, with an average age of 72.2 years. All tissuesamples were taken at the time of presentation with erythroderma and were obtained between 1996 and 1999, and only the patients for whom we had sufficient follow-up clinical information, as determined by review of the patients' charts, were included in the study.

\section{PCR and heteroduplex analysis}

Amplification and heteroduplex analysis of the TCR$\gamma$ chain was performed as previously described. ${ }^{12}$ Consensus primers for the TCR- $\gamma$ V-J junction were used to amplify genomic DNA isolated from paraffinembedded tissue. The amplification of the TCR- $\gamma \mathrm{V}$ $\mathrm{J}$ junction was performed in a Perkin-Elmer 2400 thermal cycler with a single set of consensus primers: V1 (5'-TACATCGACTGGTACGTACACGA-3') for the $\mathrm{V} \gamma 1$ family $(\mathrm{V} \gamma \mathrm{1-8})$ and $\mathrm{J} 1 / 2$ (5'-CGCGTCGACTAGGTTGGAAATGTTGTATTCTTC-3') for the $\mathrm{J} \gamma 1$ and $\mathrm{J} \gamma 2$ segments. Positive and negative controls were included in all PCR reactions.

Conditions were as follows: $100-500 \mathrm{ng}$ of genomic DNA was added to a reaction mixture containing 2.5U AmpliTaq Gold polymerase (Perkin-Elmer, Foster City, CA, USA), $10 \mathrm{pM}$ of each primer, $125 \mu \mathrm{M}$ of each dNTP, in Perkin-Elmer PCR buffer II (10 $\mathrm{mM}$ Tris-HCl, $\mathrm{pH} 8.3,50 \mathrm{mM} \mathrm{KCl}$ ) with $1.5 \mathrm{mM}$ $\mathrm{MgCl}_{2}$. After $95^{\circ} \mathrm{C}$ denaturation the TCR- $\gamma$ was amplified as follows: 20 cycles of $95^{\circ} \mathrm{C}$ denaturation $(40 \mathrm{~s}), 65^{\circ} \mathrm{C}$ annealing $(30 \mathrm{~s})$, decreasing by $1{ }^{\circ} \mathrm{C}$ / cycle, $72^{\circ} \mathrm{C}$ extension $(30 \mathrm{~s})$, followed by 15 cycles with the same denaturing and extension temperatures and an annealing temperature of $45 \mathrm{C}$, followed by a final $72^{\circ} \mathrm{C}$ extension for $5 \mathrm{~min}$.

PCR products were first visualized on a $2 \%$ agarose gel to document successful amplification. Twenty $\mu \mathrm{L}$ of the PCR product with $2 \mu \mathrm{L}$ of 0.05 M EDTA added was heated to $95^{\circ} \mathrm{C}$ for $10 \mathrm{~min}$ and then slowly cooled to $37^{\circ} \mathrm{C}$ over 30 min using the thermal cycler, then placed on ice. The samples were then electrophoresed on a Mutation Detection Enhancement (MDE) gel (FMC Bioproducts, Rocklands, ME, USA), for $12 \mathrm{~h}$ at $80 \mathrm{~V}$ in tris-borate-EDTA buffer. Heteroduplex patterns were then determined by staining with ethyidium bromide and photography on ultraviolet (UV) light.

We excluded any samples pre-dating 1996 from the study due to deterioration of the DNA. Some such samples produced a band on gel electrophoresis following $\beta$-globin PGR, performed prior to TCR- $\gamma$ PCR to check the integrity of the DNA. However, the samples did not produce bands following TCR- $\gamma$ PCR, indicating degradation of the relevant areas of DNA.

\section{Results}

\section{Clinical data}

Clinical follow-up was available for all patients in the study, and ranged from 8 to 37 months, with an average of 19 months. Of the nine men and seven women 
Heteroduplex analysis for erythroderma

Table 1. PCR Results and clinical follow-up

\begin{tabular}{|c|c|c|c|c|}
\hline patient \# & age and sex & follow-up & clinical follow-up & PCR \\
\hline 1 & $70, F$ & 37 months & drug reaction & - \\
\hline 2 & $73, \mathrm{~F}$ & 33 months & psoriasis & - \\
\hline 3 & $71, \mathrm{M}$ & 31 months & asteotic/atopic dermatitis & - \\
\hline $4 a$ & $86, M$ & 29 months & contact dermatitis & - \\
\hline $4 b$ & $86, M$ & 29 months & contact dermatitis & - \\
\hline 5 & $72, \mathrm{M}$ & 28 months & asteotic/atopic dermatitis & - \\
\hline $6 a$ & $72, \mathrm{M}$ & 18 months & contact dermatitis & - \\
\hline $6 b$ & $72, \mathrm{M}$ & 18 months & contact dermatitis & - \\
\hline $7 a$ & $56, M$ & 17 months & MF & - \\
\hline $7 \mathrm{~b}$ & $56, \mathrm{M}$ & 17 months & MF & + \\
\hline $7 \mathrm{c}$ & $56, M$ & 17 months & MF & - \\
\hline $7 d$ & $56, \mathrm{M}$ & 17 months & MF & - \\
\hline $8 a$ & $83, \mathrm{~F}$ & 17 months & contact dermatitis & - \\
\hline $8 b$ & $83, \mathrm{~F}$ & 17 months & contact dermatitis & - \\
\hline 9 & $86, F$ & 16 months & asteotic/atopic dermatitis & - \\
\hline 10 & $73, F$ & 15 months & drug reaction & - \\
\hline 11 & $79, \mathrm{~F}$ & 14 months & psoriasis & - \\
\hline $12 \mathrm{a}$ & $71, M$ & 12 months & PRP, diagnosed 12/98 & - \\
\hline $12 b$ & $71, M$ & 12 months & PRP, diagnosed 12/98 & - \\
\hline 13 & $77, \mathrm{~F}$ & 12 months & drug reaction & - \\
\hline 14 & $58, M$ & 10 months & MF/SS & + \\
\hline $15 a$ & $74, \mathrm{M}$ & 9 months & drug reaction & - \\
\hline $15 b$ & $74, \mathrm{M}$ & 9 months & drug reaction & - \\
\hline $16 a$ & $54, \mathrm{M}$ & 8 months & MF/SS & + \\
\hline \multirow[t]{4}{*}{$16 b$} & 54, M & 8 months & MF/SS & + \\
\hline & mean: 72.2 & mean: 19 mo & & \\
\hline & range: 54-86 & range:8 $-37 \mathrm{mo}$ & & \\
\hline & $9 \mathrm{M}, 7 \mathrm{~W}$ & & & \\
\hline
\end{tabular}

included in the study, three were diagnosed with MF, as determined by file review; the remaining thirteen patients' erythroderma was determined to be due to a variety of conditions: asteotic/atopic dermatitis (3 patients), generalized contact dermatitis (3 patients), drug reactions (4 patients), psoriasis (2 patients), and PRP (1 patient).

Diagnoses for the three patients with MF were based on the following: Patient \#7 had 30\% circulating Sézary cells and developed lymphadenopathy within one year after presenting with erythroderma. At the time of original presentation, however, the patient had no lymphadenopathy and no blood work was done. Patient \#14 had a history of stage la mycosis fungoides prior to presenting with erythroderma and had 30\% circulating Sézary cells and lymphadenopathy at the time of presentation with erythroderma. Patient \#16 had biopsy-confirmed involvement of an inguinal lymph node with mycosis fungoides and had $>30 \%$ circulating Sézary cells at the time of presentation with erythroderma.

Diagnoses for patients with drug reactions (patients $\# 1,10,13,15)$ were based on the observations that the patients' symptoms cleared after removal of the offending agent. Patients who were determined to have atopic dermatitis (patients \#3, 5, 9) had significant atopic histories and improved with aggressive skin emoliation and mid-strength topical steroid therapy. Patients with a diagnosis of contact dermatitis (patients \#4, 6, 8) cleared with topical and/or oral corticosteroid therapy and did not recur after avoid- ance of the offending agent. Patients \#6 and \#8 had positive reactions to allergic patch testing, and patient \#4 had a suspected source of allergy found through clinical history. The patients with psoriasis (patients \#2 and 11) both had a history of plaque-type psoriasis prior to presenting with erythroderma. Patient \#2 had mild patch-type disease for 5 years prior to developing erythroderma and continued to have typical patches of psoriasis that waxed and waned in severity after the erythroderma was treated with aggressive topical therapy. Patient \#11 was known to have a long history of severe psoriasis prior to developing erythroderma and, with improved control of her disease, she continues to have typical plaques of psoriasis. Patient \#12 had classical "islands of sparing" and had palmar-plantar hyperkeratosis and coloration typical of pityriasis rubra pilaris. Furthermore, his response to treatment with an oral retinoid was in keeping for PRP.

Table 1 summarizes the clinical data. The PCR analysis was performed in a retrospective fashion and did not play a role in establishing a final clinical diagnosis.

\section{Molecular studies}

Four of the 25 biopsies contained clonal T-cell proliferations as shown by TCR- $\gamma$ rearrangements; all four biopsies were from patients with MF. The twenty-one biopsies with polyclonal T-cell populations represent patients who, through file review, were determined to have atopic dermatitis, allergic or irritant contact dermatitis, drug reactions, PRP, psoriasis, pemphigus foliaceous, or, in one case, MF. Through serial dilutions of our positive control (DNA extracted from a lymph node with peripheral T-cell lymphoma) in our negative control (DNA extracted from tonsil), we established that a clonal T-cell population comprising less than $1 \%$ of the total DNA produced a positive result on heteroduplex analysis. Results of the TCR- $\gamma$ PCR are included in Table 1.

\section{Discussion}

The difficulty of identifying the underlying etiology of erythroderma through histopathology alone is well established, and several articles address this issue. ${ }^{1,2,}$ ${ }^{4,5}$ Walsh et al. examined 56 skin biopsies from 40 erythrodermic patients and found that the mean accuracy of the histopathological diagnoses was $53 \%$, with a range of $48-66 \%$; accuracy varied by pathologist as well as by diagnosis, e.g., the greatest degree of accuracy was observed in cases of spongiotic dermatitis and the least in those of PRP. ${ }^{4}$ Kohler et al. describe the difficulties in identifying erythrodermic MF and Sézary syndrome patients: although clearly defined histologic parameters exist for diagnoses of 


\section{Cherny et al.}

MF in well-developed patch and plaque stages, the same histologic parameters do not hold true for those patients who present with erythroderma. ${ }^{6}$ Botella-Estrada et al. also acknowledge the difficulty of assigning etiology to erythroderma through histopathology alone, citing that several biopsies were necessary before establishing a final diagnosis in eight out of a total of 36 biopsies from erythrodermic patients evaluated. Although the 36 patients collectively represented a variety of final diagnoses, of the eight patients that required multiple biopsies, five of them had CTCL, suggesting that erythrodermic CTCL has an especially challenging histopathology. ${ }^{1}$ Clearly, it would be beneficial to both patient and physician to be able to establish a diagnosis more quickly and more accurately with diminished need for multiple biopsies from each patient.

Multiple studies have evaluated the utility of PCR as a diagnostic aide in evaluating potential CTCL cases. Guitart and Kaul analyzed skin biopsies from 48 patients with "possible CTCL." Twenty-six of the 48 patients proved to have CTCL; of those 26, 19 $(73 \%)$ showed a clonal proliferation with PCR. ${ }^{10}$ Signoretti et al., in a similar study, found a clonal proliferation in 20 out of $21(95 \%)$ of T-cell lymphoma cases. $^{9}$

To the best of our knowledge, no studies have focused on the utility of TCR- $\gamma$ PCR in erythrodermic patients. With this study, we aimed to evaluate the effectiveness of establishing clonality in CTCL presenting as erythroderma. Additionally, we evaluated the potential for false-positives among the polyclonal inflammatory infiltrates associated with the majority of erythrodermic patients. In our study, 25 biopsies from sixteen patients were analyzed by TCR- $\gamma$ PGR and the results were correlated with the patients' clinical outcomes as determined through file review. Four of the 25 biopsies contained clonal TCR- $\gamma$ rearrangements; all four represented biopsies from patients who proved to have MF. These results correspond to $100 \%$ specificity and a $0 \%$ false-positive rate in this limited sample size. The remaining 21 negative TCR$\gamma$ PCR results, indicating polyclonal T-cell populations, represent thirteen different patients; 12 of these patients had inflammatory dermatoses while one patient had MF. Over the course of one month, this particular patient had four biopsies, \#7a-d, all of which were included in our study. The first two, 7a and $7 \mathrm{~b}$, were taken while the patient was on topical steroids, prescribed for the patient's ongoing papular rash of unknown etiology. The second set, $7 \mathrm{c}$ and $7 \mathrm{~d}$, were taken after the patient had discontinued his topical therapy for a period of 3 weeks. The histopathology was similarly equivocal in both sets of biopsies. During our study, one of the four biopsies produced a clonal band, whereas the remaining three did not. This not only underscores the importance of multiple biopsies, especially in patients who may be clinically suspicious for MF, but also adds weight to the presence of a positive clonal result in even one of the biopsies. It is likely that the three negative biopsies contained infiltrates that were below the threshold of detection. This patient's diagnosis was confirmed one year after the original presentation by the presence of circulating Sézary cells and lymph node involvement of T-cell lymphoma.

In conclusion, a more sensitive and specific diagnostic procedure for patients presenting with erythroderma would mean more appropriate and earlier instituted therapy, especially in the case of patients with CTCL, and avoidance of serial biopsies, particularly when the histology is ambiguous. Our study is based on a small sample of patients with a relatively short follow-up period, and similar studies with larger numbers of subjects would be necessary to substantiate our conclusions. In our hands, TCR- $\gamma$ PCR heteroduplex analysis seems to represent an important adjuvant diagnostic tool that, used in concert with histopathology and clinical history, can help to clarify underlying etiology of erythroderma.

\section{References}

1. Botella-Estrada R, Sanmartin O, Oliver V, Febrer I, Aliaga A. Erythroderma. A clinicopathological study of 56 cases. Arch Dermatol 1994; 130: 1503.

2. King LE Jr. Erythroderma. Who, where, when, why, and how. Arch Dermatol 1994; 130: 1545.

3. Kohler S, Zehnder JL. Use of the polymerase chain reaction in the evaluation of cutaneous T-cell infiltrates. Dermatol Clin 1999; 17: 657.

4. Walsh NM, Prokopetz R, Tron VA, et al. Histopathology in erythroderma: review of a series of cases by multiple observers. J Cutan Pathol 1994; 21: 419.

5. Zip C, Murray S, Walsh NM. The specificity of histopathology in erythroderma. J Cutan Pathol 1993; 20: 393.

6. Kohler S, Kim YH, Smoller BR. Histologic criteria for the diagnosis of erythrodermic mycosis fungoides and Sezary syndrome: a critical reappraisal. J Cutan Pathol 1997; 24: 292.

7. Smoller BR, Detwiler SP, Kohler S, Hoppe RT, Kim YH. Role of histology in providing prognostic information in mycosis fungoides. J Cutan Pathol 1998; 25: 311.

8. Andersen WK, Li N, Bhawan J. Polymerase chain reactiondenaturing gradient gel electrophoresis (PCR/DGGE)-based detection of clonal T-cell receptor gamma gene rearrangements in paraffin-embedded cutaneous biopsies in cutaneous T- cell lymphoproliferative diseases. J Cutan Pathol 1999; 26: 176.

9. Signoretti S, Murphy M, Cangi MG, Puddu P, Kadin ME, Loda M. Detection of clonal T-cell receptor gamma gene rearrangements in paraffin-embedded tissue by polymerase chain reaction and nonradioactive single-strand conformational polymorphism analysis. Am J Pathol 1999; 154: 67.

10. Guitart J, Kaul K. A new polymerase chain reaction-based method for the detection of T-cell clonality in patients with possible cutaneous T-cell lymphoma. Arch Dermatol 1999; 135: 158. 
11. Chhanabhai M, Adomat SA, Gascoyne RD, Horsman DE. Clinical utility of heteroduplex analysis of TCR gamma gene rearrangements in the diagnosis of T-cell lymphoproliferative disorders. Am J Clin Pathol 1997; 108: 295.
12. Kohler S, Jones CD, Warnke RA, Zehnder JL. PCR-heteroduplex analysis of T-cell receptor gamma gene rearrangement in paraffin-embedded skin biopsies. Am J Dermatopathol 2000; 22: 321 . 